\title{
Mechanism for CPT violation
}

\section{Frans R. Klinkhamer*}

Institut für Theoretische Physik, Universität Karlsruhe, D-76128 Karlsruhe, Germany E-mail: 'frans.klinkhamer@physik. uni-karlsruhe.de'

ABstract: A new mechanism for CPT violation is discussed, which relies on chiral fermions, gauge interactions and nontrivial spacetime topology. Also mentioned are the effects on the propagation of light in vacuo.

\section{Introduction}

The aim of this talk is to give a brief introduction to the so-called CPT anomaly discovered recently [i]. A more extensive review is planned for the proceedings of another conference this summer [2]. The main paper on the CPT anomaly is Ref. [i] ; further aspects have

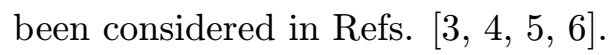

Let us start by recalling the CPT theorem due to Lüders, Pauli and Bell; see Ref. [i] and references therein. Very briefly, the "theorem" states that any local relativistic quantum field theory is invariant under the combined operation of charge conjugation $(\mathrm{C})$, parity reflection $(\mathrm{P})$ and time reversal $(\mathrm{T})$. The main inputs of this theorem are the Minkowski spacetime, the invariance under transformations of the restricted Poincaré group $\mathcal{P}_{+}^{\uparrow}$, the normal spin-statistics connection and, finally, the locality and hermiticity of the Hamiltonian; cf. Ref. [i]

The following question now arises: can CPT invariance be violated at all in a physical theory and, if so, is it in the real world? It is clear that something "unusual" is required for this to be the case.

Two possibilities, in particular, have been discussed in the literature. The first is quantum gravity, which may or may not lead to CPT violation; cf. Ref. "8in. The point is, of course, that Poincaré invariance does not hold in general. Still, a CPT theorem can be "proven" quite generally for asymptotically-flat Euclidean spacetimes $\left[\begin{array}{l}\overline{9} \\ \underline{9}\end{array}\right]$. The second possibility is superstring theory, which may or may not lead to CPT violation; cf. Ref. [i] 10 ]. The point, now, is the (mild) nonlocality of the theory. But, to date, no convincing calculation has shown the necessary violation of CPT.

\footnotetext{
${ }^{*}$ Speaker.
} 
Here, we report on a simple mechanism for $\mathrm{CPT}$ violation, which does not require the introduction of radically new (but unproven) theories. It turns out, namely, that for certain chiral gauge field theories and spacetime topologies, CPT invariance is broken by quantum effects [i]

\section{CPT anomaly: an example}

The CPT anomaly is best illustrated by a concrete example. That is, we take the chiral gauge field theory with group $G$ and left-handed fermion representation $R_{L}$ given by

$$
\left(G, R_{L}\right)=(S O(10), \mathbf{1 6}+\mathbf{1 6}+\mathbf{1 6}),
$$

defined over the spacetime manifold $\mathrm{M}$ with metric g given by

$$
(\mathrm{M}, \mathrm{g})=\left(\mathbb{R}^{3} \times S^{1}, \eta_{\mu \nu}^{\text {Minkowski }}\right),
$$

with coordinates

$$
x^{0} \equiv c t, x^{1}, x^{2} \in \mathbb{R} \quad \text { and } \quad x^{3} \in[0, L] .
$$

Then there is necessarily CPT violation, with a typical mass scale [i, $\left[\begin{array}{l}1 \\ 1\end{array}\right]$

$$
M_{G} \equiv \frac{\alpha_{G} \hbar}{L c} \sim 10^{-35} \mathrm{eV}\left(\frac{\alpha_{G}}{0.01}\right)\left(\frac{210^{10} \mathrm{lyr}}{L}\right)
$$

where $\alpha_{G} \equiv g^{2} /(4 \pi)$ is defined in terms of the dimensionless $S O(10)$ gauge coupling constant $g$ and $L$ is the size of the compact dimension.

We also observe that the CPT anomaly appears for the $S O(10)+\left(\mathbf{1 6}_{L}\right)^{3}$ theory over the $\mathbb{R}^{3} \times S^{1}$ manifold, but neither for $S O(10)+\left(\mathbf{1 6}_{L}\right)^{3}$ over $\mathbb{R} \times S^{3}$ nor for QED over $\mathbb{R}^{3} \times S^{1}$. Hence, $\underline{\text { both }}$ topology and parity violation are crucial ingredients of the CPT anomaly; see Section 5 of Ref. [i]

As regards the role of topology, the CPT anomaly resembles the Casimir effect, with the local properties of the vacuum depending on the boundary conditions; cf. Ref. [i]1i]. In fact, the CPT anomaly will be seen to make the vacuum optically active.

Clearly, it is important to be sure of this surprising effect and to understand better the underlying mechanism. Some progress has been made for two-dimensional theories in particular.

\section{CPT anomaly in 2 dimensions}

Consider, then, chiral $U(1)$ gauge theory over the torus $T^{2} \equiv S^{1} \times S^{1}$, with a Euclidean metric $g_{\mu \nu}(x)=\delta_{\mu \nu} \equiv \operatorname{diag}(1,1)$. Concretely, we take the gauge-invariant model with five left-handed fermions of charges $(1,1,1,1,-2)$. Henceforth, we set $\hbar=c=1$.

In this case, the effective Euclidean action $\Gamma[a]$ for the $U(1)$ gauge field $a_{\mu}(x)$, defined by the path integral

$$
\exp (-\Gamma[a]) \equiv \int \prod_{f=1}^{5}\left(\mathcal{D} \bar{\psi}_{R f} \mathcal{D} \psi_{L f}\right) \exp \left(-S\left[\bar{\psi}_{R f}, \psi_{L f}, a\right]\right),
$$


is known exactly. For doubly-periodic boundary conditions on the spinors in particular, the effective action $\Gamma_{\mathrm{PP}}[a]$ is given in terms of Riemann theta functions.

It is now straightforward to verify that a CPT transformation of the $U(1)$ gauge field $a_{\mu}(x)$ does not leave this effective action invariant:

$$
\exp \left(-\Gamma_{\mathrm{PP}}[a]\right) \rightarrow-\exp \left(-\Gamma_{\mathrm{PP}}[a]\right), \quad \text { for } \quad a_{\mu}(x) \rightarrow-a_{\mu}(-x) .
$$

Hence, the $1111 \overline{2}$-model over $T^{2}$ with doubly-periodic boundary conditions unambiguously

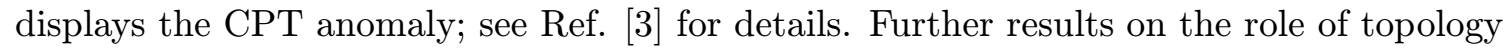
and torsion are given in Ref. [葪]

\section{CPT anomaly in $3+1$ dimensions}

Let us return to $3+1$ dimensions and take, again, the $S O(10)$ chiral gauge theory $\left(\begin{array}{l}2 \\ 2\end{array}-1,1\right)$ over the cylindrical spacetime manifold $\mathrm{M}=\mathbb{R}^{3} \times S^{1}$. The metric has Lorentzian signature, $g_{\mu \nu}(x)=\eta_{\mu \nu} \equiv \operatorname{diag}(-1,1,1,1)$. Note that this particular chiral gauge theory contains the Standard Model with three families of quarks and leptons.

The effective action $\Gamma[A]$, for $A \in \operatorname{so}(10)$, is, of course, not known exactly. But the crucial term has been identified perturbatively [i]1]

$$
\Gamma_{\mathrm{CS}-\text { like }}^{\mathbb{R}^{3} \times S^{1}}[A]=\int_{\mathbb{R}^{3}} \mathrm{~d} x^{0} \mathrm{~d} x^{1} \mathrm{~d} x^{2} \int_{0}^{L} \mathrm{~d} x^{3} \frac{n \pi}{L} \omega_{\mathrm{CS}}\left[\vec{A}\left(\vec{x}, x^{3}\right)\right],
$$

with the Chern-Simons density

$$
\omega_{\mathrm{CS}}[\vec{A}] \equiv \frac{1}{16 \pi^{2}} \epsilon^{k l m} \operatorname{tr}\left(F_{k l} A_{m}-\frac{2}{3} A_{k} A_{l} A_{m}\right)
$$

in terms of the Yang-Mills field strength $F_{k l} \equiv \partial_{k} A_{l}-\partial_{l} A_{k}+\left[A_{k}, A_{l}\right]$. The Latin vector indices $k, l, m$, in Eq. (1)

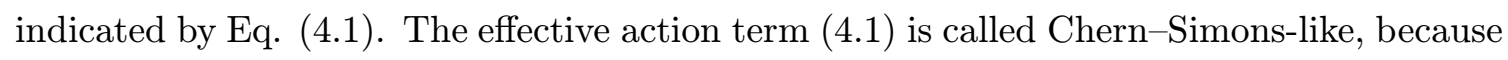
a genuine topological Chern-Simons term exists only in an odd number of dimensions.

The integer $n$ in Eq. ( by

$$
n=\sum_{f=1}^{3}\left(2 k_{0 f}+1\right), \quad k_{0 f} \in \mathbb{Z} .
$$

Since the sum of an odd number of odd numbers is odd, one has $n \neq 0$, which implies the

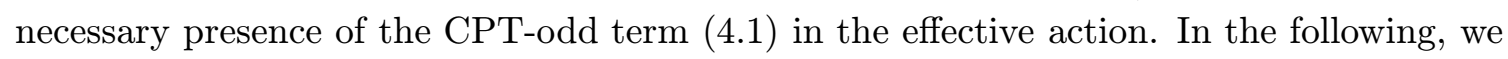
consider the simplest case

$$
n=(1-1+1) \Lambda_{0} /\left|\Lambda_{0}\right|= \pm 1,
$$

where $\Lambda_{0}$ is an ultraviolet Pauli-Villars cut-off for the $x^{3}$-independent modes of the fermionic fields contributing to the effective action.

Focusing on the $U(1)$ gauge field $a_{\mu}(x)$ embedded in $A_{\mu}(x) \in \mathrm{so}(10)$, we have the following quadratic terms in the effective Lagrangian density:

$$
\mathcal{L}_{\mathrm{MCS}}[a]=-\frac{1}{4} \eta^{\kappa \mu} \eta^{\lambda \nu} f_{\kappa \lambda} f_{\mu \nu} \mp \frac{1}{4} M \epsilon^{3 \kappa \lambda \mu} f_{\kappa \lambda} a_{\mu},
$$




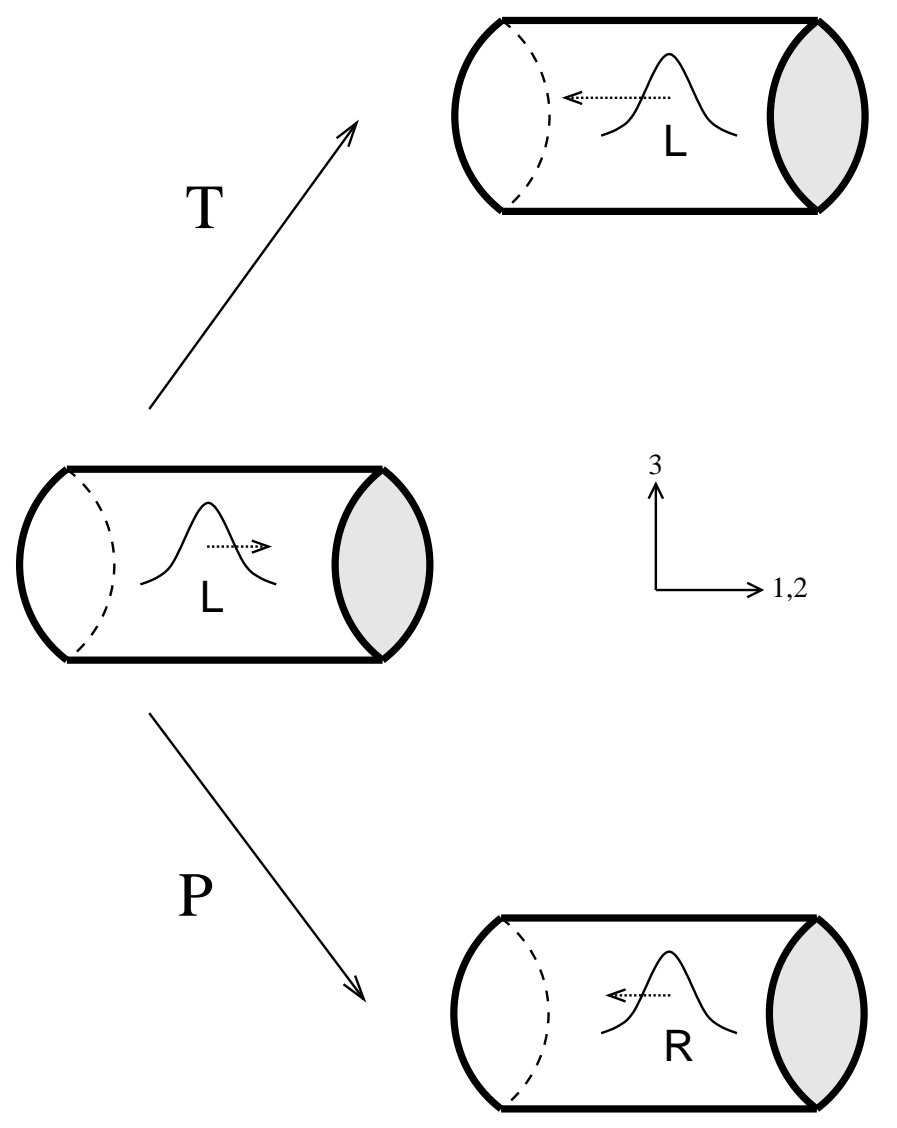

Figure 1: Sketch of the behavior of a left-handed wave packet in the MCS theory (4.5i) under time reversal $(\mathrm{T})$ and parity $(\mathrm{P})$ transformations, the charge conjugation $(\mathrm{C})$ transformation acting trivially. The dotted arrows indicate the group velocity approximately in the $x^{1}$ or $x^{2}$ direction, for the case of a compact $x^{3}$ coordinate. The magnitude of the group velocity changes under $\mathrm{T}$, but not under $\mathrm{C}$ or $\mathrm{P}$ (hence, the physics is CPT-noninvariant). Also, the vacuum is seen to be optically active, with left- and right-handed light pulses traveling to the left at different speeds.

with the Maxwell field strength $f_{\mu \nu} \equiv \partial_{\mu} a_{\nu}-\partial_{\nu} a_{\mu}$ and Chern-Simons parameter $M \sim \alpha / L$. Note that the action corresponding to the Chern-Simons-like term in Eq. ( $\overline{4} . \overline{5} . \bar{j})$ is gauge invariant, provided the electric and magnetic fields in $f_{k l}$ vanish fast enough as $|\vec{x}| \rightarrow \infty$.

Two aspects of the Maxwell-Chern-Simons (MCS) theory (4. detail [is] field theory. ${ }^{1}$ Second, the photon propagation makes clear that $\mathrm{C}$ and $\mathrm{P}$ are conserved, but $\mathrm{T}$ not. In fact, one has for the group velocity of a left-handed electromagnetic pulse propagating approximately in the "orthogonal" direction $\left(x^{2}\right.$, say):

$$
\left|\vec{v}_{\mathrm{g}}^{L}\left(0, p_{2}, \delta p_{3}\right)\right| \neq\left|\vec{v}_{\mathrm{g}}^{L}\left(0,-p_{2},-\delta p_{3}\right)\right|,
$$

\footnotetext{
${ }^{1}$ For a timelike Chern-Simons-like term, with $\epsilon^{3 \kappa \lambda \mu}$ in Eq. (14.5. not hold, as long as unitarity is imposed [5]. This result may also have some bearing on the question whether or not a Chern-Simons-like term can be radiatively induced from a CPT-violating axial-vector term in the Dirac sector. It appears not [6.;.
} 
for $0<M \ll 2 \pi / L \ll\left|\delta p_{3}\right| \ll\left|p_{2}\right|$. This result shows the time-reversal noninvariance for a concrete physical situation (recall that momentum changes sign under $\mathrm{T}$, whereas helicity remains unchanged). See Fig. 1 for a sketch of the effect and Ref. [i2] for further details.

\section{Conclusions}

The subtle role of topology on the local properties of quantum field theory is well-known (e.g. the Casimir effect). For chiral gauge theories such as the Standard Model, this may also lead to CPT noninvariance, even for flat spacetime manifolds, i.e. without gravity [i]1. Potential applications of the CPT anomaly include:

- the optical activity of the vacuum, as shown in Fig. 1 (leading to a polarization effect

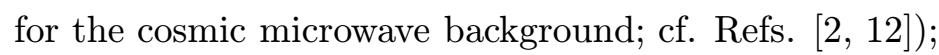

- the fundamental arrow-of-time (possibly playing a role at the beginning of the uni-

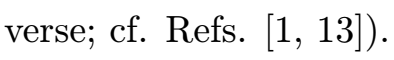

A further interesting property of the four-dimensional CPT anomaly is the connection between the ultraviolet and the infrared, as shown by the factors $n$ and $1 / L$ in the effective

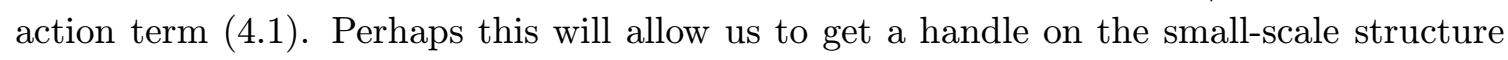
of spacetime (wormholes, ... ) by studying the long-range behavior of photons.

\section{Acknowledgments}

The author thanks the organizers of the conference and the parallel session for the opportunity to present his results. He also thanks C. Mayer for help with the figure.

\section{References}

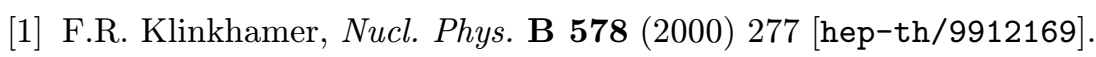

[2] F.R. Klinkhamer, in: Y.S. Kim and M.E. Noz (Eds.), Proceedings y-th International Wigneri i symposium [hep-th/0110135.

[3] F.R. Klinkhamer and J. Nishimura, Phys. Rev. D 63.2001$)$ 0977011 hep-th/0006154].

[4] F.R. Klinkhamer and C. Mayer, Nucl. Phys. B 616_2001) 215i [hep-th/0105310].

[5] C. Adam and F.R. Klinkhamer, 'Nucl. Phys. B 607 (2001) $2 \overline{4} \overline{7}$ [hep-ph/0101087].

[6] C. Adam and F.R. Klinkhamer,

[7] G. Lüders, Ánn. Phys. $(N \bar{Y}) \mathbf{2}(1957) 1_{1}^{\prime}$.

[8] R. Wald, 'Phys. Rev. D 21 (1980) 2742

[9] L. Alvarez-Gaumé and C. Gomez,

[10] V.A. Kostelecký and R. Potting, iNucl. Phys. B 359

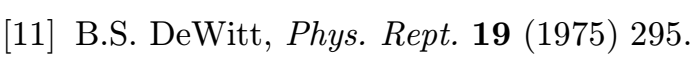

[12] N.F. Lepora, [gre qc/9812077il].

[13] R. Penrose, in: S.W. Hawking and W. Israel (Eds.), General Relativity: An Einstein Centenary Survey, Cambridge University Press 1979. 